\title{
A SPURIOUS PULSE PROBLEM IN A GAS FLOW PROPORTIONAL COUNTER
}

\author{
Daniel J. GRADY and J. Craig ROBERTSON * \\ Department of Nuclear Engineering, The University of Michigan, Ann Arbor, Michigan 48109, USA
}

Received 9 June 1980

The source of spurious pulses in a $4 \pi \beta$ gas flow proportional counter was traced to impurities in the counting gas. The concentration of the impurities was so small that it was not obvious that this was the cause of the problem. It is suggested that the long term degradation in gas flow detectors may be due to the accumulation on the anode wires of microscopic deposits from the counting gas.

\section{Introduction}

One aspect of the nuclear data acquisition program currently underway in our department is the absolute measurement of neutron capture cross sections using foil activation techniques. One of the most efficient devices for the detection of beta activity is a $4 \pi$ gas flow proportional counter. A detector of this type was built, based on the design of Campion [1], but during its initial operation an unusual spurious pulse problem was encountered. Spurious pulses were observed from the counter even when no source was present. Both the amplitude of the pulses and their rate increased with the time of operation of the counter. It is the purpose of this short paper to discuss the cause of this problem so that other experimenters may avoid the frustrations we experienced.

\section{The source of the spurious pulses in a gas flow proportional counter}

We immediately suspected that the source of the problem was in our method of mounting the central anode wires of the counter, even though this closely followed the methods used by others. It seemed very probable that electrical breakdown must be the cause of the problem. However, after many attempts to remedy the situation by changing the design used in

\footnotetext{
* Present address: Department of Chemical and Nuclear Engineering, The University of New Mexico, Albuquerque, New Mexico 87131, USA.
}

mounting our anode wires, it was realized that this was not the source of the problem and we turned our attention to the P-10 gas used in the detector. Chemi$\mathrm{cal}$ analysis had failed to find any impurity. Nevertheless, when we observed our anode wires under a microscope, it was clear that microscopic deposits were being formed and that the source of these must be trace concentrations of contaminants in the counter gas.

\section{The cause of the problem}

Anode wires which had been operated at $1950 \mathrm{~V}$ for one week, as well as a control sample of the wire, were examined with a scanning electron microscope (SEM). Fig. 1 is an electron micrograph of the control wire at a magnification of $760 \mathrm{X}$. This wire, never used in the detector, was perfectly free from deposits of any sort.

Quite the opposite is true of the wires used in the detector and exposed to the P-10 gas. The two micrographs in figs. 2 and 3 demonstrate the interaction of a component of the gas with a component of the anode wire (made of phosphor-bronze). The dendritic deposit on the wire in fig. 2 (380X magnification) appears to follow a die line from the drawing of the wire. This suggests that the growth of the deposits initiated at surface defects on the wire and supports the possibility that the interaction is chemical in nature.

Figure 3 (1900X magnification) is a micrograph of the wire surface beneath a deposit which was inten- 


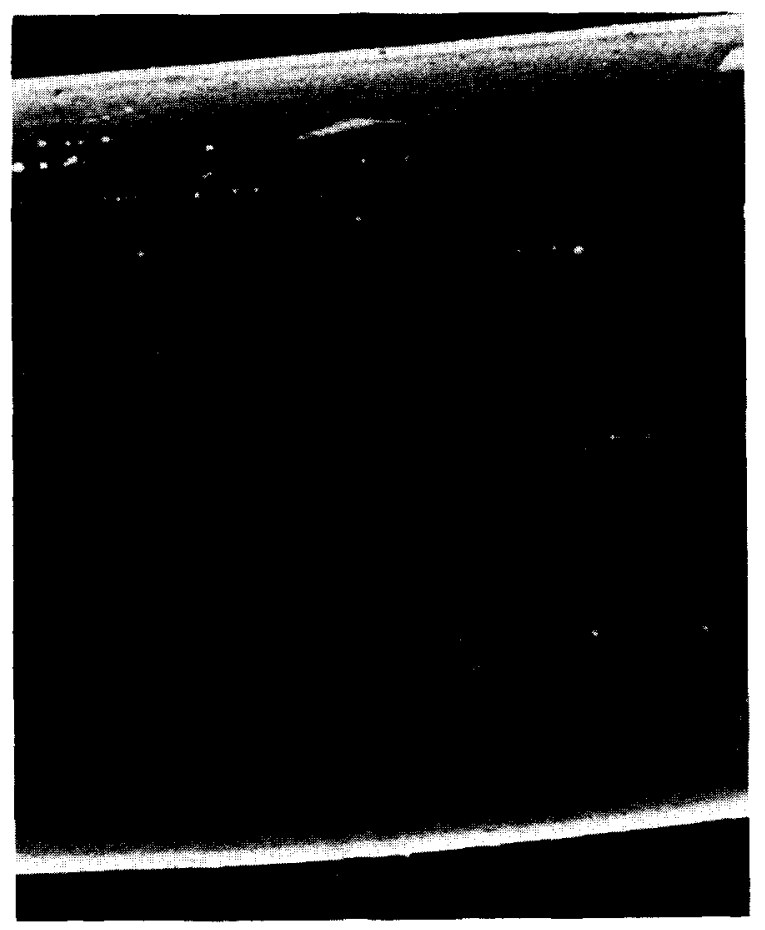

Fig. 1. Electron micrography of a control wire at $760 \times$ magnification shows no remarkable surface defects or deposits.

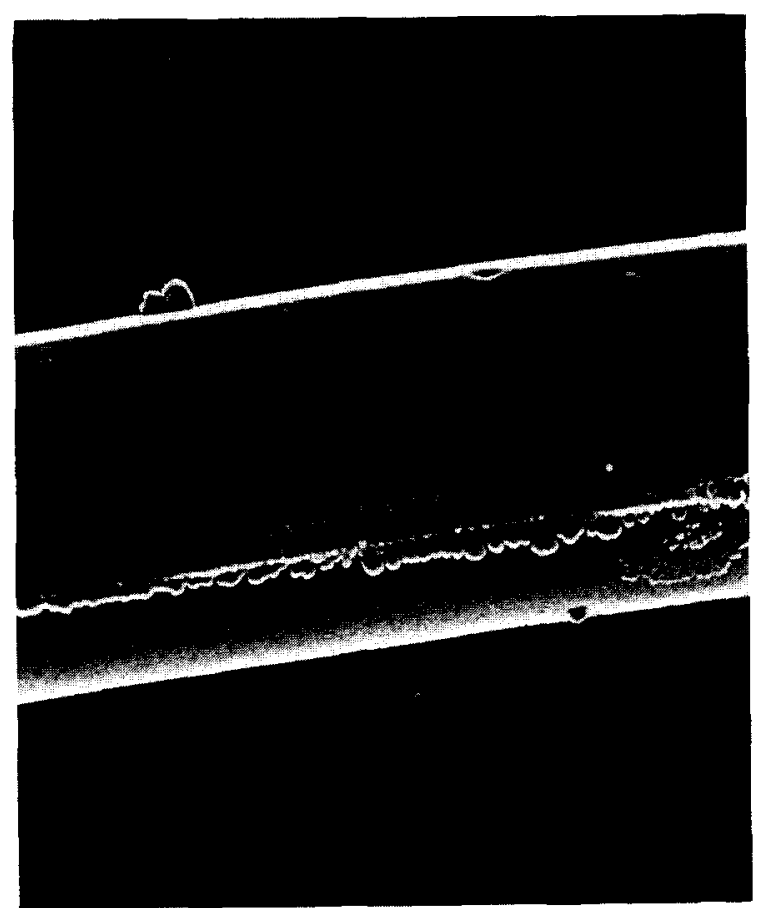

Fig. 2. Electron micrograph of a portion of the anode exposed to the gas and operated at $1950 \mathrm{~V}$ for one week. Magnification of $380 \times$ shows dendritic deposit along axis of wire apparently along die line.

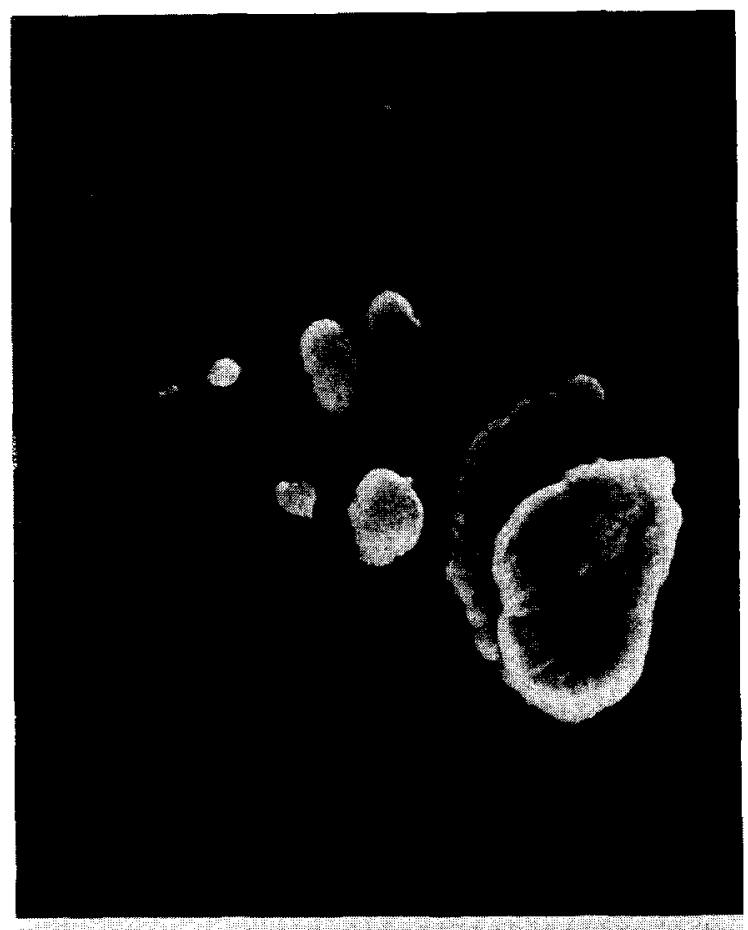

Fig. 3. Electron micrograph of the wire surface beneath an intentionally removed portion of the deposit at $1900 \times \mathrm{mag}-$ nification. The exaggerated die lines and the smooth, deep pit are characteristic of chemical attack on the wire surface.

tionally removed. Both the smooth pit and the deep, exaggerated die lines observed in this photograph are also characteristic of the results of chemical attack, further substantiating the chemical interaction theory.

Satisfied that the spurious pulse effect was caused by the deposits produced from the chemical interaction of a gas contaminant with the wire, we next determined the deposit composition. Using a scanning electron microprobe the characteristic X-rays of the deposit were excited by an electron beam, detected by a thin window proportional counter and identified by their energies. In all of the deposits examined, the composition was predominantly copper (component of the wire) and sulfur (apparent component of the P-10 gas contaminant). Additional proof that the deposits were indeed copper and sulfur (probably copper sulfide) was provided by both copper and sulfur X-ray imaging. The source of the X-rays coincided exactly with the location of the deposits on the wire. 
Based upon the results of this investigation, it appears that a low level, chemically reactive, sulfur bearing contaminant in the P-10 gas was the cause of the spurious pulse problem observed in the gas flow proportional counter. The concentration of the contaminant was, however, very small. A gas chromatography-mass spectrometer analysis with a detection threshold of $0.05 \%$ revealed no contaminant.

\section{Spurious pulse formation}

During the initial investigation of the deposits with the SEM, several deposits exhibited image flickering

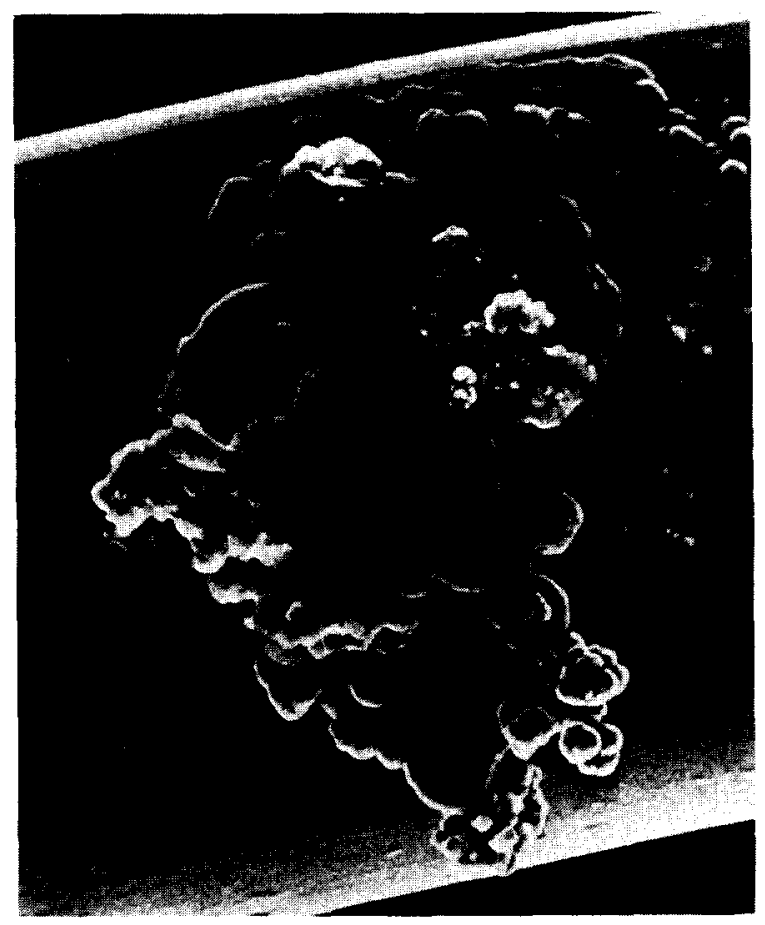

Fig. 4. Electron micrograph of a large deposit at $2000 \times$ magnification shows evidence of surface charging. Bright areas of the deposit are locations of electron buildup from the SEM beam. This phenomenon suggests that the spurious pulse mechanism may be like a shorting insulator. on the CRT display of the SEM. This effect is common in non-conductive samples which accumulate electric charge from the electron beam. On the micrograph of fig. 4 (760X magnification) the effect is manifested by relatively bright spots on the photograph. This charging effect suggests a potential mechanism for the formation of the spurious pulses.

It is possible that the copper sulfide deposits act as insulators. Electrons produced by ionizing events in the detector and multiplied by the avalanche effect could accumulate on these deposits until enough charge existed to allow a short to the anode. The resulting pulse would depend upon factors such as deposit thickness and resistance, but the pulse could certainly be mistaken as a true signal pulse.

\section{Conclusion}

Specifically, we can conclude that low level, sulfur bearing contaminants in the P-10 counting gas which chemically interacted with the anode metal, led to spurious pulse formation in the proportional counter. Although the fill gas is perhaps not normally considered a likely source of difficulty in gas-filled radiation detectors, this study would recommend its consideration when detector response deviates from expected performance. Much frustration might be avoided.

It is possible that the long term degradation in performance of gas flow detectors may be due, at least in part, to the accumulation of very low concentration contaminants (in the counter gas) on the anode wires.

This work was sponsored by the Department of Energy as part of the on-going work in absolute cross section measurements at the University of Michigan in the Department of Nuclear Engineering.

\section{References}

[1] P.J. Campion, Int. J. Appl. Rad. Isot. 4 (1959) 232. 